J. Clin. Chem. Clin. Biochem.

Vol. 15, 1977, pp. 561-564

\title{
Serumcholinesterase als Modell eines Glykoproteins
}

\author{
Von G. Uhlenbruck $\left.{ }^{1}\right)$, H. Haupt, I. Reese und G. Steinhausen \\ Mediz inische Universitätsklinik Köln-Lindenthal und Behringwerke AG, Marburg
}

(Eingegangen am 7. April/20. Juni 1977)

Zusammenfassung: Die Cholinesterase (EC 3.1.1.8) im Serum des Menschen stellt ein kohlenhydratreiches Glykoprotein dar, welches mit 18 verschiedenen Lectinen aus Pflanzen und Invertebraten in Form einer spezifischen PräzipitinReaktion reagiert; die meisten davon erfassen alkalistabil gebundene Kohlenhydratketten. Ein Drittel dieser Lectin-Rezeptoren erscheint erst nach Neuraminidase-Behandlung, zwei Drittel können vor und nach Entfernung der Neuraminsäure so demonstriert werden. Die spezifischen Lectin-Rezeptoren der alkali-labilen Kohlenhydratketten werden charakterisiert und analysiert, und zwar mit chemischen und serologischen Methoden.

\section{Serum cholinesterase as a model glycoprotein}

Summary: Human serum cholinesterase (EC 3.1.1.8) is a carbohydrate-rich glycoprotein, which reacts with 18 different lectins from plants and invertebrates by a specific precipitin reaction; most of the lectins combine with alkali-stable bound carbohydrate chains. One third of these lectin receptors appear after neuraminidase-treatment, two thirds can be demonstrated before and after removal of neuraminic acid. The specific lectin receptors of the alkali-labile carbohydrate chains are characterized and analyzed by chemical and serological methods.

\section{Einleitung}

Die Cholinesterase (EC 3.1.1.8) des Humanserums ist ein Glykoprotein, das zu etwa 24\% aus Kohlenhydraten und zu etwa $76 \%$ aus Aminosäuren besteht $(1,2)$. Sie besitzt ein Molekulargewicht von 348000 und ist aus vier Untereinheiten aufgebaut $(2,3)$. Ihr Gehalt im Humanserum beträgt knapp $10 \mathrm{mg} / 1$. Von klinischer Bedeutung ist der genetisch bedingte Polymorphismus der Serumcholinesterase im Zusammenhang mit ihrer Fähigkeit, das in der Anästhesie verwendete Muskelrelaxans Succinylbischolinchlorid (Suxamethoniumchlorid) abzuzubauen $(4,5,6)$.

Die vorliegende Arbeit beschäftigt sich mit drei wesentlichen Aspekten des Kohlenhydratanteils der Serumcholinesterase:

1. Es wird die Präzipitinreaktion dieses Enzyms mit verschiedenen Lectinen getestet und zwar vor und nach Neuraminidase-Behandlung.

2. Serologisch wird mit Hilfe dieser Lectine das Vorkommen eines kryptantigenen A-like Antigens sowie des T-Rezeptors. (Thomsen-Friedenreich) festgestellt.

1) Mit Unterstützung der Deutschen Forschungsgemeinschaft.
3. Beide Rezeptoren konnten gaschromatographisch identifiziert werden, da sie als alkali-labile Kohlenhydratketten vorkommen.

\section{Material und Methoden}

Lectine

Die in dieser Arbeit benutzten Lectine sind bereits ausführlich in einer vorhergehenden Mitteilung beschrieben worden (7).

Immunfiffusion

Die Durchfuihrung erfolgte ebenfalls wie bereits andernorts beschrieben (7).

Serumcholinesterase

Hochgereinigtes Präparat nach Haupt et al. (1).

Disaccharid-A nalyse

Die Entfernung der Neuraminsäure durch milde Säurehydrolyse sowie die ạnschließende Alkali-Borhydrid Behandlung wurde nach Newman et al. (8) durchgefürt. Eine zweite Probe wurde ohne Abspaltung der Neuraminsäure in gleicher Weise behandelt.

\section{Silylierung}

Diese erfolgte nach der Methode von Sweeley et al. (9), wobei allerdings fự die Reaktion Hexamethyldisilazan und Trimethylchlorosilan als Gemisch verwendet wurden.

Die gaschromatographische Identifikation des Disaccharids geschah mit Hilfe des isothermen Programms bei $250^{\circ} \mathrm{C}$ mit einem 
$\mathrm{N}_{2}$-Durchfluß von $45 \mathrm{ml} / \mathrm{min}$. Durchführung der Gaschromatographie nach Newman et al. (8).

Bestimmung von

alkalilabil gebundenem $\mathrm{N}$-A cetyl-galakto samin

Diese wurde analog der Disaccharidanalyse durchgeführt, wobei das N-Acetylgalaktosamin durch die Alkali-Borhydrid-Behandlung und nachfolgende Säurehydrolyse in Galaktosaminit übergeht, das im Gaschromatographen nachgewiesen wird bei einem Temperaturprogramm von $125^{\circ}-230^{\circ} \mathrm{C}$ mit einer Steigerung von $4^{\circ} \mathrm{C}$ pro min. Interner Standard: anstelle von Trehalose Erythrit.

\section{Monosaccharid-Bestimmung}

Diese Analyse erfolgte ebenfalls gaschromatographisch. Die Methode wurde in einer vorhergehenden Arbeit eingehend beschrieben (8).

\section{Ergebnisse}

Präzipitation von Cholinesterase durch verschiedene Lectine

Das Ergebnis dieser Experimente ist in der Tabelle 1 festgehalten. Zunächst einmal können wir zwei Hauptgruppen unterscheiden: Während die Invertebraten-Lectine ausschließlich nach Entfernung der Neuraminsäure reagieren, und hierhin gehört sensu strictu auch das AxinellaLectin, präzipitieren die pflanzlichen Lectine alle auch schon vorher.

Als Ėrgebnis der Vèrsuche mit Invertebraten-Lectinen können wir sagen, daß das Vorkommen von $\beta-D$-Galaktosyl-Strukturen, wahrscheinlich in $\beta(1-4)$-glykosidischer Bindung an N-Acetyl-D-glucosamin, nach Abspaltung der Neuraminsäure als gesichert gelten kann; dafür sprechen auch die Versuche mit pflanzlichen Lectinen, deren Reaktion anschließend verstärkt war.

Ferner kommen $\alpha$-N-Acetyl-galaktosaminyl (sogen. Blutgruppen A-like)-Strukturen heraus, wie die Versuche - mit Ausnahme allerdings von Cepaea nemoralis - mit den Lectinen von Schnecken zeigen. Es ist bekannt, daß solche Strukturen alkali-labil an Hydroxyaminosäuren gebunden sind.

Die pflanzlichen Lectine lassen sich hier in drei Gruppen einteilen: Einmal solche, welche mit endständiger oder mittelständiger $D$-Galaktose (Rutilus) reagieren und welche das Vorhandensein von $\beta-D$-Galaktosido-

Tab. 1. Reaktion von verschiedenen Lectinen mit Serum-Acetylcholinesterase.

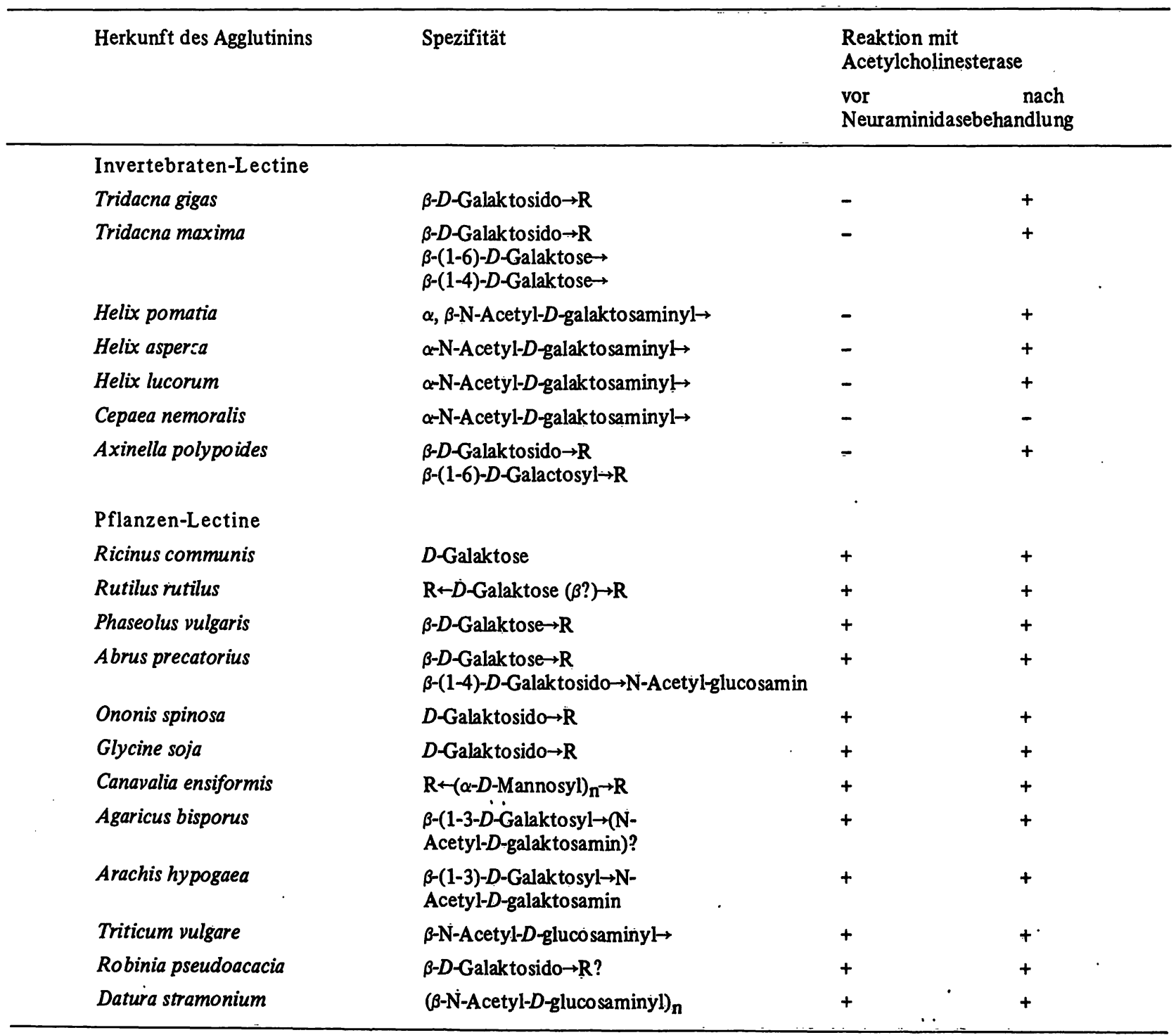


Resten vor und nach Neuraminidase-Behandlung anzeigen. Hierzu gehören insbesondere die Lectine aus Ricinus, Phaseolus, Abrus, Ononis, Glycine soja und Robinia.

Eine zweite Gruppe erfaßt typische alkali-stabile Kohlenhydratketten (10), hierzu rechnet man Concanavalin A (Con A), die Lectine aus Datura stramonium und Triticum, das Weizenkeimlectin. Sie reagieren mit typischen Zuckern alkali-stabiler Ketten: $D$-Mannose und N-Acetyl$D$-glucosamin.

Als letzte Gruppe sind die Arachis/Agaricus-Lectine zu erwähnen. Sie reagieren, und das gilt insbesondere für das Arachis-Lectin, mit der alkali-labilen DisaccharidStruktur, die wir als T-Antigen im Sinne von Thomsen \& Friedenreich identifizieren konnten (8). Als ,reines“ Anti-T kann man nur das Arachis-Lectin betrachten, während das Agaricus-Lectin auch mit alkali-stabilen Ketten reagieren könnte (10). Bemerkenswert ist, daß dieses $T$ Antigen, zumindest teilweise, auch ohne Abspaltung der Neuraminsäure zugänglich ist, also frei sein muß.

Die Präzipationsteste sind anhand eines Beispiels in Abbildung 1 aufgezeigt. Es fällt auf, daß sich mit Ricinus und Glycine soja Doppellinien ergeben. Ferner reagieren die Lectine aus Ononis und $A$ brus mit einem unterschiedlichen Rezeptor (Spornbildung). Bemerkenswert ist auch, daß Concanavalin A aus der Pflanze Canavalia ensiformis sowohl mit dem gereinigten Ricinus-Lectin, als auch mit Phaseolus-Lectin reagiert, ein oft zu erhebender Befund, der auf die Glykoproteinnatur der Lectine selbst hinweist. In Abbildung 2 ist demonstriert, daß verschiedene andere Glykoproteine, getestet mit einem dieser Lectine, Identitätslinien mit der Serumcholinesterase ergeben, was in diesem Fall auf sehr ähnliche Kohlenhydratstrukturen schließen läßt (nach Wintzer, Vaith \& Uhlenbruck, noch unveröffentlicht).

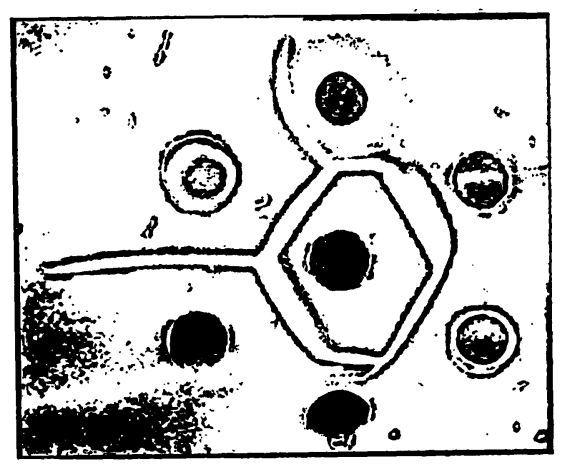

Abb. 1. Präżipitationsreaktionen verschiedener Lectịne pflanzlicher Herkunft mit Neuraminidase-behandelter Serumcholinesterase vom Menschen.

Numerierung im Uhrzeigersinn von $12 \mathrm{Uhr}$ an.

Mitte $=$ Neuraminidase-behandelte Cholinesterase $1=$ Ricinus communis

$2=$ Glycine soja

$3=$ Ononis spinosia

$4=$ Abrus precatorius

$5 \doteqdot$ Phaseolus vulgaris (PHA)

$6=$ Concanavalin $A$ (Con A)

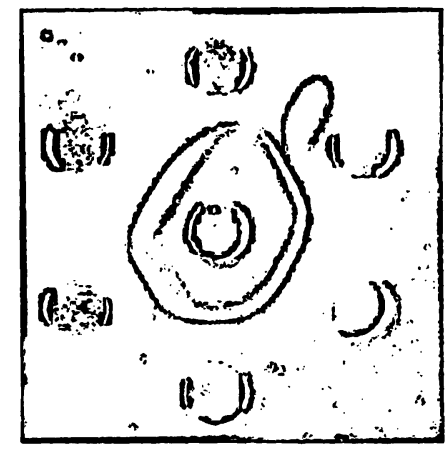

Abb. 2. Präzipitationsreaktionen mehrerer Glykoproteine, inklusive Serumcholinesterase, mit einem dieser Lectine (Agaricus bisporus, Mitte).
$1=$ carcinoembryonales Antigen
2 = Mördermuschel Hämolymphe (Tridacna gigas)
3 = Hummermagen-Glykoprotein
$4=$ Glykoprotein aus Kaviareiern
$5=$ Serumcholinesterase (Mensch)
$6=$ Blutgruppensubstanz A aus Schweinemagen

Kohlenhydrat-A nalysen der Serumcholinesterase

\section{Monosaccharide}

Die Analyse der Monosaccharide ergab folgende Werte in Gewichtsprozent: Fucose $(0,2 \%)$, Mannose $(3,1 \%)$, Galaktose (3,5\%), Galaktosamin (4,2\%), Glucosamin $(8,8 \%)$. Durch den hohen Glucosamingehalt wird auch die starke Reaktion von Serumcholinesterase mit den Lectinen aus Triticum vulgare und Datura stramonüm verständlich.

\section{Alkali-labil gebundenes $N$-Acetyl-galaktosamin}

Dieses wurde zu 1,6\% bezogen auf das Gesamtmolekül, bestimmt. Dies erklärt zwar die Reaktion der Helix-Präzipitine, aber auch, warum nicht alle gleich stark diesen Rezeptor anzeigten. Aufgrund der serologischen und gaschromatographischen Analyse müssen wir annehmen, daß eine alkali-labil gebundene N-Acetyl-neuraminylN-Acetyl-D-galaktosaminyl-Kette im Molekül der genuinen Serumcholinesterase vorliegen muß (an Serin oder Threonin gebunden).

\section{Disaccharid-Analyse (T Antigen)}

Das Disaccharid $\beta$-D-Galaktosido-(1-3)-N-Acetyl-D-galaktosamin kommt, in freier Form und auch durch Neuraminsäure blockiert, zu 4,28\%, bezogen auf das Gewicht des Gesamtmoleküls, vor. Die freie Form des Disaccharids wurde zu $0,23 \%$ bestimmt, d. h. 5,31\% des GesamtDisaccharids ist frei von Neuraminșäureresten.

Auch diese Kohlenhydratgruppe ist alkali-labil über Hydroxyaminosäuren (Serin, Threonin) an die Proteinkette gebunden. 


\section{Diskussion}

Die vorliegende Arbeit charakterisiert das Enzym Serumcholinesterase als Modell eines Glykoproteins. Es besitzt zwei alkali-labile Ketten, von denen eine, nämlich das "A-like" Antigen, $\alpha$-N-Acetyl-D-galaktosaminyl $\rightarrow$ Protein (Serin, Threonin), durch Neuraminsäure blockiert ist.

Die zweite alkali-labile Kette, welche aus dem Disaccharid $\beta$-D-Galaktosyl-(1-3)-N-Acetyl-D-galaktosamin ( $\rightarrow$ Protein) besteht, liegt teilweise in freier Form vor, ist aber auch durch Neuraminsäure blockiert. Beide Ketten hàben sich erst serologisch und dann gaschromatographisch nachweisen lassen.

Darüber hinaus gibt es endständige, freie $\beta-D$-GalactosylGruppen, die sehr wahrscheinlich Bestandteil einer oder mehrerer alkali-stabiler Kohlenhydratketten sind. Letztere werden durch die Reaktion der Lectine von Canavalia ensiformis, Triticum vulgare, Datura stramonium und Abrus precatorius bzw. Phaseolus vulgaris angezeigt (10).

Diese Lectine insgesamt (Tab. 1) ermöglichen nicht nur eine Reinigung des Glykoproteins, sondern auch das Studium der Hetèrógenität einzelner Rezeptoren bzw. von Isoenzym-Varianten, wie dies in der schematischen Abbildung 3 dargestellt ist. Sie eröffnen ferner die Möglichkeit, das Enzym zu binden, ohne das enzymatisch aktive Zentrum zu beeinflussen, oder aber die Wirkung des Ënzyms durch sterische Beeinträchtigung des aktiven Zentrums zu blockieren oder abzuschwächen. Experimente in dieser Richtung dürften weiteren Aufschlußૂ über die Topographie von Kohlenhydratanteil(en) einerseits und dem aktiven Zentrum andererseits ergeben.

\section{Danksagung}

Wir danken den Herren $V$. Hořejší, $A$. Krajhanzl und $J$. Köcourek (Prag), O. Prokop (Berlin), R. Newman und B. P. Chatterjee (Köln) für die Überlassung hochgereinigter Lectin-P̣räparate.

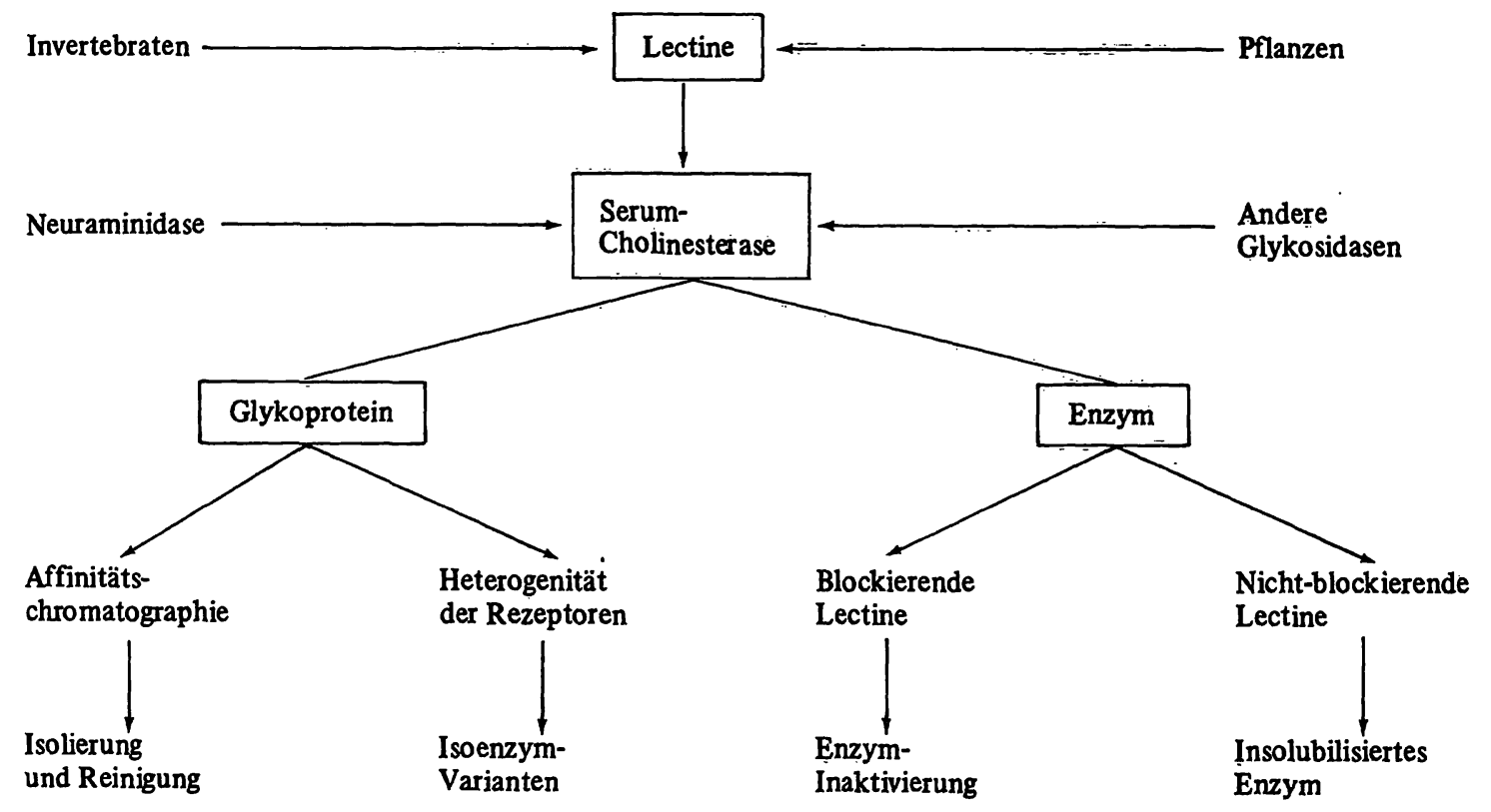

Abb. 3. Wechselwirkungen zwischen dem Glykoprotein Cholinesterase, dem Enzym Cholinesterase und verschiedenen Lectinen.

\section{Literatur}

1. Haupt, H., Heide, K., Zwisler, O. \& Schwick, H. G. (1966), Blut 14, 65-76.

2. Schwick, H. G., Heide, K. \& Haupt, H. in "Glycoconjugates" (M. J. Horowitz, ed.), Vol. I, Chap. 4, Acad, Press (im Druck).

3. Scott, E. N. \& Powers, R. F. (1972), Nature 236, 83-84.

4. Doenicke, A., Schmidinger, St. \& Kruzney, I (1968); Brit.

J. Anaesth. 40, 834-844.

5. Günther, R., Dönhardt, A., Altland, K., Jensen, M. \&

Goedde, H. W. (1971), Med. Klinik 66, 785-788.

6. Happle, R., Scholler, K. L. \& Münscher, H. (1973), Anaesthesist 22, 224-226.
7. Uhlenbruck, G., Steinhausen, G., Schwick, H. G., Hořejší, V., Krajhanzl, A., Kocourek, J. \& Chatterjee, B. P. Biochim. Biophys. Acta. (im Druck).

8. Newman, R. A., Harrison, R. \& Uhlenbruck, G. (1976), Biochim. Biophys. Acta 433, 344-356.

9. Sweeley, C. C., Bentley, R., Maketā, M. \& Wells, W. W. (1963), J. Am. Chem. Soc̣. 85, 2497-2507.

10. Uhlenbruck, G., Newman, R. A., Steinhausen, G. \& Schwick, H. G. (1977), Z. Immun.-Forsch. 153, 183-187.

Prof. G. Uhlenbruck

Abt. Immunbiologie

Kerpener Straße 15

5000 Köln 41

W. Germany 